\title{
The role of ramucirumab and pembrolizumab combination in patients with advanced non-small cell lung cancer, gastroesophageal adenocarcinoma, or urothelial carcinoma
}

\author{
Jane E. Rogers ${ }^{1}$, Jaffer A. Ajani ${ }^{2}$ \\ ${ }^{1}$ Pharmacy Clinical Programs, U.T. M.D. Anderson Cancer Center, Houston, TX, USA; ${ }^{2}$ Department of Gastrointestinal Medical Oncology, U.T. \\ M.D. Anderson Cancer Center, Houston, TX, USA \\ Correspondence to: Jaffer A. Ajani, MD. U.T. M.D. Anderson Cancer Center, 1515 Holcombe Blvd, Houston, TX 77030, USA. \\ Email: jajani@mdanderson.org. \\ Comment on: Herbst RS, Arkenau HT, Santana-Davila R, et al. Ramucirumab plus pembrolizumab in patients with previously treated advanced non- \\ small-cell lung cancer, gastro-oesophageal cancer, or urothelial carcinomas (JVDF): a multicohort, non-randomised, open-label, phase 1a/b trial. \\ Lancet Oncol 2019;20:1109-23.
}

Submitted Apr 01, 2020. Accepted for publication May 30, 2020.

doi: $10.21037 /$ cco-20-132

View this article at: http://dx.doi.org/10.21037/cco-20-132

Antibody against vascular endothelial growth factor ligand (anti-VEGF) or receptor (anti-VEGFR) and antibody against programmed death-1 (anti-PD-1) receptor or programmed death ligand 1 (anti-PD-L1) are often not combined to treat patients with advanced malignancies. Recent proposals have postulated that such a combination could produce synergistic effect thus providing greater clinical advantage than realized otherwise (1). Herbst et al. recently reported an anti-VEGFR and anti-PD-1 combination for three advanced solid tumor cohorts: gastroesophageal adenocarcinoma (GEA), non-small cell lung cancer (NSCLC), and urothelial carcinoma (UC) (2). Such patients have considerable unmet needs and novel approaches are needed (3-5).

Currently, ramucirumab, anti-VEGFR, has been approved to treat advanced GEA and NSCLC patients in the second line setting but it produces minimum advantage $(6,7)$. Ramucirumab's limited role in NSCLC patients has been criticized (8). In GEA patients, the combination of paclitaxel and ramucirumab is the preferred regimen (3) over marginal effects of ramucirumab alone $(3,6,9)$. The checkpoint inhibition (IO) immunotherapy with pembrolizumab was approved for $3^{\text {rd }}$ or later line therapy of advanced GEA patients based solely on the response rate but 4 randomized studies have failed to produce survival benefit from IO $(3,10-13)$. Thus we can say with certainty that advanced GEA is barely responsive to IO and most patients have an evidence of cancer progression at the time of first response assessment.

However, the scenario is quite different for NSCLC and UC where IO therapy has made considerable impact in patient outcome (14-18). The role of IO therapy in advanced NSCLC depends on the absence of the targetable driver mutations (4). Patients with tumors without these driver mutations are candidates for anti-PD-1 or antiPD-L1 therapy alone or in combination with platinum doublet chemotherapy. Anti-PD-1 therapy preference for monotherapy vs combination with chemotherapy is dependent on the level (labeling index) of PD-L1 expression. Pembrolizumab monotherapy is preferred for those with PD-L1 expression $\geq 50 \%$ while using pembrolizumab in combination with platinum doublet therapy is for those with PD-L1 1-49\%. Responses seen in KEYNOTE-024 were approximately $45 \%$ with pembrolizumab and a clear advantage over platinum doublet chemotherapy (4 months improvement in PFS) in this group (17). Subsequent therapy options for those that did not receive an anti-PD-1 or anti-PD-L1 agent in the front-line may receive one of these options depending on specific indication (4). Interesting to note, bevacizumab has been studied in combination with atezolizumab plus chemotherapy (ABCP regimen) compared to bevacizumab plus chemotherapy. The advantage was with the ABCP regimen showing $63.5 \%$ response and median PFS 
8.3 months (16). The ABCP regimen, however, is not the preferred National Comprehensive Cancer Network (NCCN) front-line regimen currently, likely due to the lack of understanding of the contribution of anti-VEGF along with safety concerns (4). The regimen is a front-line option for those without targetable driver mutations and regardless of PD-L1 expression levels.

For advanced urothelial carcinoma, single agent IO therapy (pembrolizumab, nivolumab, atezolizumab, avelumab, durvalumab) is reserved second-line following cisplatin combination therapy failure regardless of PDL1 status (5). Additionally, for those patients ineligible for cisplatin in the front-line, atezolizumab or pembrolizumab are considered alternative regimens front-line. Responses seen in the second-line setting with anti-PD-1 or antiPD-L1 therapy are roughly 20\% (15-25\%) with a slight potential advantage for those with PD-L1 positive UCs. Pembrolizumab compared to second-line chemotherapy in platinum refractory front-line patients showed a $21 \%$ response rate and median PFS of 2.1 months (14). AntiVEGF therapy currently is not approved or incorporated in advanced UC management; however, the use of these agents has been investigated alone or in combination with a chemotherapy backbone $(5,19)$. Unfortunately, most results have been disappointing.

The interplay between tumor cells and the immune system is made up of multiple complex processes. One of the well-known mechanisms of resistance to IO is upregulation of the angiogenic pathway $(1,20)$. High intratumor VEGF levels hinder $T$ cell infiltration through increased interstitial fluid pressure and creating a hypoxic microenvironment (1). In addition, high VEGF levels promote immune inhibitory cells, such as regulatory T-cells and immature myeloid suppressor cells, and suppress dendritic cell, antigen presenting cells, and maturation. Therefore, theoretically, there is good rationale to block both the immune checkpoint(s) and angiogenic pathway $(1,20)$. The advantage of this approach is realized in patients with hepatocellular carcinoma (21).

The Herbst et al. report combined ramucirumab plus pembrolizumab in advanced GEA (one to two lines of previous therapy), NSCLC (one to three lines of previous therapy), and UC (one to three lines of previous therapy) patients (2). Patients with GEA or UC had not received a prior anti-angiogenic agent or IO agent. While NSCLC patient were exposed to VEGF or VEGFR, although the percentage of these patients was not reported. Forty-one patients were included in the GEA cohort, 27 patients in the NSCLC cohort, and 24 patients in the UC cohort. Rare grade 4 toxicities were seen. Twenty-four percent had grade 3 or more toxicities in the entire population mostly being hypertension and colitis. One patient in the GEA cohort died of pulmonary sepsis that was deemed to be treatment-related. Overall, the combination appears safe without unexpected toxicities. The question is whether this combination provides any benefit? The data are promising for the NSCLC and UC cohorts: For NSCLC patients, a $30 \%$ response rate was observed with a median progressionfree survival (PFS) of 9.7 months. Considering, $41 \%$ of tumors were PD-L1 negative and $15 \%$ had a PD-L1 expression of $1-49 \%$, this combination warrants a further look. The data are borderline in the UC group, with a $13 \%$ response rate and 1.9-month median PFS. Here we do not feel enthusiastic about pursuing this combination. However, the results are disappointing in the GEA cohort. Therefore, we disagree with the conclusion in the report that this combination is active in patients with GEA. The observed response rate with only $7 \%$ and the median PFS was 4.6 months.

There are several trials reporting similar combinations in patients with advanced solid tumor (22). Hara et al. reported the combination of nivolumab and ramucirumab in 46 patients in the second-line GEA setting (22). The median PFS was $\sim 3$ months and the response rate was $26.7 \%$. Such discrepant results reflect patient heterogeneity, small number of patients in a single-arm study (16). Hironaka et al. reported the outcomes of 43 advanced GEA patients treated with ramucirumab plus paclitaxel plus nivolumab with a response rate of $37.2 \%$ and the median PFS of 5.8 months (23).

The landscape for IO therapy is dynamic as we identify new ways to overcome resistance. We encourage continued translational research to determine ways in which each solid tumor differs with regard to resistance along with pressing forward with agents that target IO barriers. Some strategies of overcoming resistance currently underway include immune checkpoint inhibitors plus other checkpoint inhibitors, immune stimulatory agents (i.e, anti-OX40), metabolic modulators (i.e, IDO inhibitors), immune modulators (i.e, TGF $\beta$ inhibitors), macrophage inhibitors, vaccines, targeted therapies (i.e, anti-MEK; anti-EGFR), radiation therapy, chemotherapy, epigenetic modifications (i.e, histone deacetylase inhibitors), NK activation, cell therapies among others (20). 


\section{Acknowledgments}

Funding: None.

\section{Footnote}

Provenance and Peer Review: This article was commissioned by the editorial office, Chinese Clinical Oncology. The article did not undergo external peer review.

Conflicts of Interest: Both authors have completed the ICMJE uniform disclosure form (available at http://dx.doi. org/10.21037/cco-20-132). Dr. Ajani reports that he has received research funds from Merck and he is also a paid advisor to Merck. The other author has no conflicts of interest to declare.

Ethical Statement: The authors are accountable for all aspects of the work in ensuring that questions related to the accuracy or integrity of any part of the work are appropriately investigated and resolved.

Open Access Statement: This is an Open Access article distributed in accordance with the Creative Commons Attribution-NonCommercial-NoDerivs 4.0 International License (CC BY-NC-ND 4.0), which permits the noncommercial replication and distribution of the article with the strict proviso that no changes or edits are made and the original work is properly cited (including links to both the formal publication through the relevant DOI and the license). See: https://creativecommons.org/licenses/by-nc-nd/4.0/.

\section{References}

1. Yi M, Jiao D, Qin S, et al. Synergistic effect of immune checkpoint blockade and anti-angiogenesis in cancer treatment. Mol Cancer 2019;18:60.

2. Herbst RS, Arkenau HT, Santana-Davila R, et al. Ramucirumab plus pembrolizumab in patients with previously treated advanced non-small-cell lung cancer, gastro-oesophageal cancer, or urothelial carcinomas (JVDF): a multicohort, non-randomised, open-label, phase 1a/b trial. Lancet Oncol 2019;20:1109-23.

3. Network, N.C.C. Gastric cancer. Available online: https:// www.ncen.org

4. Network, N.C.C. Non-Small Cell Lung Cancer. Available online: https://www.ncen.org

5. Network, N.C.C. Bladder Cancer. Available online: https://www.nccn.org

6. Fuchs CS, Tomasek J, Yong CJ, et al. Ramucirumab monotherapy for previously treated advanced gastric or gastro-oesophageal junction adenocarcinoma (REGARD): an international, randomised, multicentre, placebocontrolled, phase 3 trial. Lancet 2014;383:31-9.

7. Garon EB, Ciuleanu TE, Arrieta O, et al. Ramucirumab plus docetaxel versus placebo plus docetaxel for secondline treatment of stage IV non-small-cell lung cancer after disease progression on platinum-based therapy (REVEL): a multicentre, double-blind, randomised phase 3 trial. Lancet 2014;384:665-73.

8. Mok TS, Loong HH. A REVELation in non-small-cell lung cancer treatment? Lancet 2014;384:640-2.

9. Wilke H, Muro K, Van Cutsem E, et al. Ramucirumab plus paclitaxel versus placebo plus paclitaxel in patients with previously treated advanced gastric or gastro-oesophageal junction adenocarcinoma (RAINBOW): a double-blind, randomised phase 3 trial. Lancet Oncol 2014;15:1224-35.

10. Tabernero J, Van Cutsem E, Bang YJ, et al. Pembrolizumab with or without chemotherapy versus chemotherapy for advanced gastric or gastroesophageal junction (G/GEJ) adenocarcinoma: The phase III KEYNOTE-062 study. J Clin Oncol 2019;37:LBA4007.

11. Shitara K, Özgüroğlu M, Bang YJ, et al. Pembrolizumab versus paclitaxel for previously treated, advanced gastric or gastro-oesophageal junction cancer (KEYNOTE-061): a randomised, open-label, controlled, phase 3 trial. Lancet 2018;392:123-33.

12. Bang YJ, Ruiz EY, Van Cutsem E, et al. Phase III, randomised trial of avelumab versus physician's choice of chemotherapy as third-line treatment of patients with advanced gastric or gastro-oesophageal junction cancer: primary analysis of JAVELIN Gastric 300. Ann Oncol 2018;29:2052-60.

13. Moehler MH, Dvorkin M, Ozguroglu M, et al. Results of the JAVELIN Gastric 100 phase 3 trial: avelumab maintenance following first-line (1L) chemotherapy $(\mathrm{CTx})$ vs continuation of CTx for HER2- advanced gastric or gastroesophageal junction cancer (GC/GEJC). J Clin Oncol 2020;38:abstr 278.

14. Bellmunt J, de Wit R, Vaughn DJ, et al. Pembrolizumab as Second-Line Therapy for Advanced Urothelial Carcinoma. N Engl J Med 2017;376:1015-26.

15. Balar AV, Galsky MD, Rosenberg JE, et al. Atezolizumab as first-line treatment in cisplatin-ineligible patients with locally advanced and metastatic urothelial carcinoma: a single-arm, multicentre, phase 2 trial. Lancet 


\section{Page 4 of 4}

2017;389:67-76.

16. Socinski MA, Jotte RM, Cappuzzo F, et al. Atezolizumab for First-Line Treatment of Metastatic Nonsquamous NSCLC. N Engl J Med 2018;378:2288-301.

17. Reck M, Rodríguez-Abreu D, Robinson AG, et al. Pembrolizumab versus Chemotherapy for PD-L1Positive Non-Small-Cell Lung Cancer. N Engl J Med 2016;375:1823-33.

18. Gandhi L, Rodríguez-Abreu D, Gadgeel S, et al. Pembrolizumab plus Chemotherapy in Metastatic NonSmall-Cell Lung Cancer. N Engl J Med 2018;378:2078-92.

19. Narayanan S, Srinivas S. Incorporating VEGF-targeted therapy in advanced urothelial cancer. Ther Adv Med

Cite this article as: Rogers JE, Ajani JA. The role of ramucirumab and pembrolizumab combination in patients with advanced non-small cell lung cancer, gastroesophageal adenocarcinoma, or urothelial carcinoma. Chin Clin Oncol 2021;10(3):30. doi: 10.21037/cco-20-132
Rogers and Ajani. Ramucirumab plus pembrolizumab combination

Oncol 2017;9:33-45.

20. Sharma P, Hu-Lieskovan S, Wargo JA, et al. Primary, Adaptive, and Acquired Resistance to Cancer Immunotherapy. Cell 2017;168:707-23.

21. Network, N.C.C. Hepatocellular Carcinoma. Available online: https//www.nccn.org

22. Hara H, Shoji H, Takahari D, et al. Phase I/II study of ramucirumab plus nivolumab in patients in second-line treatment for advanced gastric adenocarcinoma (NivoRam study). J Clin Oncol 2019;37:abstr 129.

23. Hironaka S, Kadowaki S, Izawa N, et al. A phase I/II study of nivolumab, paclitaxel, and ramucirumab as second-line in advanced gastric cancer. J Clin Oncol 2020;38:abstr 352. 